\title{
OPEN miRNAs of Aedes aegypti (Linnaeus 1762) conserved in six orders of the class Insecta
}

\author{
Iram Pablo Rodríguez-Sanchez ${ }^{1,10 \bowtie}$, Daniel Rafael Saldaña-Torres ${ }^{1,10}$, \\ Olga Karina Villanueva-Segura ${ }^{1}$, Maria Lourdes Garza-Rodriguez ${ }^{2}$, Mayra A. Gómez-Govea ${ }^{1}$, \\ Ghongwei Liang ${ }^{3}$, María de Lourdes Ramírez-Ahuja ${ }^{1}$, Margarita De La Luz Martinez-Fierro ${ }^{4}$, \\ Ivan Delgado-Enciso ${ }^{5,6}$, Laura E. Martinez-de-Villarreal ${ }^{7}$, Yu Zhou ${ }^{3}$, Adriana E. Flores-Suarez ${ }^{8}$, \\ Xi Chen ${ }^{3}$, Diana Resendez-Pérez ${ }^{9}$, Chen-Yu Zhang ${ }^{3}$ \& Gustavo Ponce-Garcia ${ }^{8 凶}$
}

Aedes aegypti $\mathrm{L}$. is the most important vector of arboviruses such as dengue, Zika, chikungunya, Mayaro, and yellow fever, which impact millions of people's health per year. MicroRNA profile has been described in some mosquito species as being important for biological processes such as digestion of blood, oviposition, sexual differentiation, insecticide resistance, and pathogens dissemination. We identified the miRNAs of Ae. aegypti females, males and eggs of a reference insecticide susceptible strain New Orleans and compared them with those other insects to determine miRNA fingerprint by new-generation sequencing. The sequences were analyzed using data mining tools and categorization, followed by differential expression analysis and conservation with other insects. A total of 55 conserved miRNAs were identified, of which 34 were of holometabolous insects and 21 shared with hemimetabolous insects. Of these miRNAs, 32 had differential expression within the stages analyzed. Three predominant functions of miRNA were related to embryonic development regulation, metamorphosis, and basal functions. The findings of this research describe new information on Ae. aegypti physiology which could be useful for the development of new control strategies, particularly in mosquito development and metamorphosis processes.

Diptera is a major order that includes disease vectors of importance to human health. The mosquito Aedes aegypti (L.) belongs to the family Culicidae, one of the most dangerous to public health because the large number of diseases that it transmits to humans, such as dengue, Zika, yellow fever, chikungunya, and Mayaro viruses ${ }^{1}$. Disease dissemination occurs due to the effective invasive characteristics of Ae. aegypti such as the drying resistant eggs, and other independent physical factors such as climate change and urban sprawl ${ }^{2}$. Molecular physiology investigation of these organisms could lead to a better understanding in viral dissemination and adaptive ecology of vector insects.

MicroRNA (miRNA) is a small sequence of RNA ( 22 nucleotides in length), which has genetic regulatory functions in eukaryotes ${ }^{3}$. These molecules contain within structure a region called seed, responsible for the hybridization specificity with a target transcript; the region size is 6 nucleotides (nt) and starts in the second

\footnotetext{
${ }^{1}$ Universidad Autonoma de Nuevo Leon, Facultad de Ciencias Biologicas, Laboratorio de Fisiología Estructural y Molecular, Av. Universidad S/N CD. Universitaria, 66455 San Nicolas de los Garza, Nuevo Leon, Mexico. ${ }^{2}$ Universidad Autónoma de Nuevo León, Hospital Universitario "Dr. José Eleuterio González", Centro Universitario Contra el Cáncer., Monterrey, Nuevo Leon, Mexico. ${ }^{3}$ State Key Laboratory of Pharmaceutical Biotechnology, NJU Advanced Institute of Life Sciences, Jiangsu Engineering Research Center for MicroRNA Biology and Biotechnology, Nanjing University, Nanjing 210093, China. ${ }^{4}$ Laboratorio de Medicina Molecular, Universidad Autonoma de Zacatecas, Unidad Academica de Medicina Humana, 98160 Zacatecas, Zacatecas, Mexico. ${ }^{5}$ Facultad de Medicina, Universidad de Colima, 28040 Colima, Colima, Mexico. ${ }^{6}$ Instituto Estatal de Cancer, Secretaria de Salud de Colima, Colima, Mexico. ${ }^{7}$ Universidad Autonoma de Nuevo Leon, Facultad de Medicina, Departamento de Genética, 64460 Monterrey, Nuevo Leon, Mexico. ${ }^{8}$ Universidad Autonoma de Nuevo Leon, Facultad de Ciencias Biologicas, Departamento de Zoologia de Invertebrados, Laboratorio de Entomologia Medica, Av. Universidad S/N CD. Universitaria, 66455 San Nicolas de los Garza, Nuevo Leon, Mexico. ${ }^{9}$ Universidad Autonoma de Nuevo Leon, Facultad de Ciencias Biologicas, Departamento de Biología Celular y Genética, 66455 San Nicolás de Los Garza, Nuevo Leon, México. ${ }^{10}$ These authors contributed equally: Iram Pablo Rodríguez-Sanchez and Daniel Rafael Saldaña-Torres. ${ }^{\square}$ email: iramrodriguez@gmail.com; gustavo.poncegc@uanl.edu.mx
} 


\begin{tabular}{|l|c|r|l|}
\hline Stage & Total reads & \multicolumn{1}{l|}{ Mapped reads } & miRNAs read with a miRDeep2 (score $\geq 4)$ reported for Aedes aegypti \\
\hline Eggs & $4,474,188$ & $1,691,089(\sim 38 \%)$ & $16,198\left(66^{*}\right)$ \\
\hline Females & $9,409,689$ & $4,613,825(\sim 49 \%)$ & $14,490\left(72^{*}\right)$ \\
\hline Males & $7,748,704$ & $4,017,274(\sim 52 \%)$ & $10,127\left(61^{*}\right)$ \\
\hline Total & $21,632,581$ & $10,322,188(\sim 48 \%)$ & $40,815\left(80^{*}\right)$ \\
\hline
\end{tabular}

Table 1. miRNA annotation by biological stage. \% Correspond to the number of reads recovered with respect to their total number in each of the approaches. ${ }^{*}$ Correspond to the number of miRNAs previously reported to Ae. aegypti in miRDeep2.

nucleotide in the $5^{\prime}$ to $3^{\prime}$ direction ${ }^{4}$. Ae. aegypti (like many other organisms) has a physiologically fundamental relation with different miRNAs. Some miRNAs have been experimentally validated, including those that manage blood digestion and metamorphosis processes such as aae-miR-1890 ${ }^{5}$, sexual dimorphism regulation such as aae-miR- $309^{6}$, viral interactions such as aae-miR- $375^{7}$ or bacterial diseases as in the case of aae-miR- $12^{8}$ and aae-miR-2940-5 $\mathrm{p}^{9}$. On the other hand, other miRNAs have been found silencing more than one target, allowing the complex physiological phenomena regulation for example, aae-miR-1174 which regulates diverse intestinal functions-sugar absorption, fluid excretion and blood intake ${ }^{10}$-or aee-miR-275 involve in blood digestion processes $^{11}$

Within the organisms of the class Insecta, miRNAs are molecules that have undergone constant alterations. This has revealed that miRNA conservation rates in insects are very low. An example of this miRNA conservation is that Tribolium Castaneum Herbst 1797 and Drosophila melanogaster Meigen 1830 have only a third of the total miRNAs conserved between them ${ }^{12}$.

Mosquitos conserve a large number of miRNAs such as miR-281, miR-184, miR-989, and miR-278, which are shared with Ae. aegypti, Culex quinquefasciatus Say 1832 and Anopheles gambiae Giles $1902^{13}$. Even so, there are miRNAs that are unique to every species as in the case of aae-miR-2946 for Ae. aegypti or cqu-miR-2951 and cqu-miR-2952 for Cx. quinquefasciatus ${ }^{14}$.

This is the first report of miRNA differential expression in comparison with other insects and three life stages of Ae. aegypti. The objective of this study was to describe the miRNA fingerprint of Ae. aegypti by Next Generation Sequencing. This information is especially relevant as it can be a powerful tool for the proposal of new strategies for targeted species control without affecting ecologically important organisms.

\section{Results}

miRNAs annotation. A total of 21,632,581 reads were obtained; 40,815 showed similarity in the structure and biogenesis of 80 different miRNAs of Aedes aegypti. The number of readings for each biological stage is provided in Table 1.

miRNAs taxonomic distribution. Of the 80 miRNAs annotated from Ae. aegypti, 55 showed significant conservation with 713 miRNAs from other insect species, while 25 showed Ae. aegypti-specific conservation, considering as cut-off an E value $\leq 0.005$. According to the NCBI taxonomy information of these insect species, we identified that of the 713 miRNAs, 445 miRNAs (62.41\%) were present in the order Diptera, 130 in Hymenoptera (18.23\%), 84 in Lepidoptera (11.78\%), 29 in Coleoptera (4.07\%), 19 in Hemiptera (2.66\%) and 6 in Orthoptera (0.84\%) as described in Fig. 1 and Supplementary Material 1.

Tendency of Ae. aegypti miRNAs conservation in taxonomic orders. Of the 55 conserved miRNAs, 15 conservation patterns were obtained in the six orders previously mentioned (x-axis, Fig. 2A). We found specifically for Diptera 16 miRNAs conserved (y-axis in bar graph form, Fig. 2A). Also, of the 55 conserved miRNAs, 34 were only found in holometabolous insect orders (Diptera, Lepidoptera, Coleoptera and Hymenoptera) and 21 miRNAs were shared with hemimetabolous orders-Hemiptera and Orthoptera (Fig. 2B); none of miRNAs was unique to hemimetabolous orders.

miRNA presence in females, males and eggs. Figure 3 showed 55 conserved miRNAs: 4 showed the specific distribution in females (miR-1175-5p, miR-193, miR-285 and miR-31), 3 in males (miR-263a-5p, miR71-5p and miR-993) and 4 in eggs (miR-2944a-5p, miR-307, miR-996 and miR-33). Of the 44 remaining miRNAs, 7 were found in females and eggs, 7 in males and eggs, 1 in females and males, and 29 miRNAs were shared among females, males and eggs (Supplementary Material 2).

Differential expression of miRNAs in three life stages and conserved in the class Insecta. LRT was used to analyze the 44 miRNAs that showed expression in more than one life stage; among these, we found that only 21 miRNAs exhibited differential expression (DE) in the three contrasts (Fig. 4A). These were considered downregulated or upregulated, depending on their statistical significance $(L R>4$ and $p$ value $\leq 0.01)$ and fold change behavior (negative or positive) in comparison to their mean expression among the three life stages (Supplementary Material 2). A total of 17 miRNAs were downregulated; of these, five were in males (bantam5p, miR-286b, miR-375, miR-7, miR-9c-5p), seven in females (miR-10, miR-100, miR-184, miR-282-5p, miR305-5p, miR-92b-3p, miR-965) and five in eggs (miR-125-5p, miR-1891, miR-2765, miR-932-5p, let-7). We also 


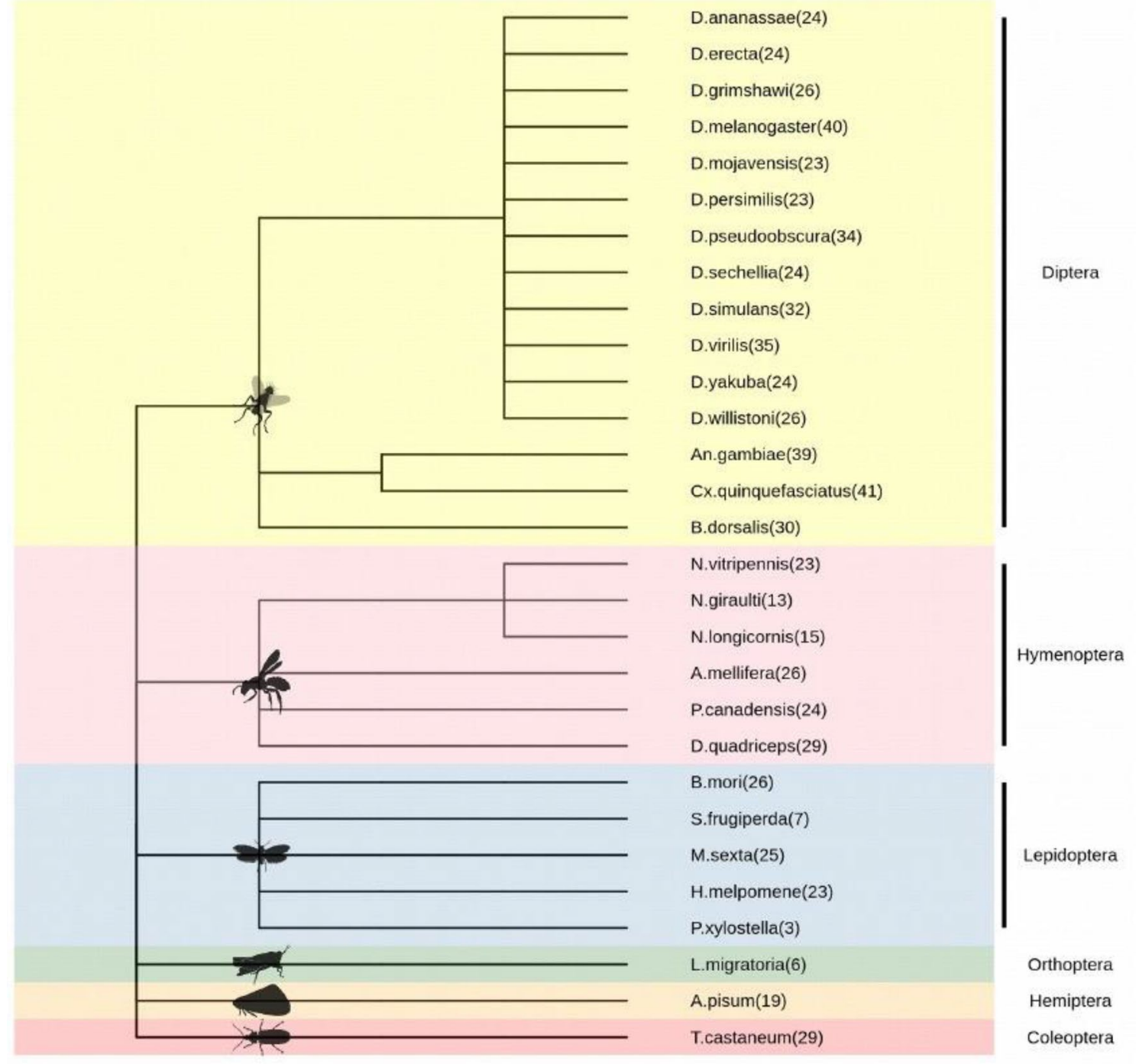

Figure 1. Taxonomic distribution of the 713 conserved miRNAs in class Insecta and their abundance by species.

identified four upregulated miRNAs, one in females (miR-989) and two in eggs (2944b-5p, miR-iab-4-5p); one miRNA was present in both females and eggs (miR-2941).

Of the 21 miRNAs with DE found in females, males and eggs of Ae. aegypti have been found in six orders of Insecta as shown in Fig. 4B: in Diptera, eight miRNAs were specific (bantam-5p, miR-1891, miR-286b, miR-2941, miR-2944-5p, miR-9c-5p, let-7, miR-989). In all the holometabolous, three miRNAs were conserved (miR-282-5p, miR-305-5p, miR-932-5p) and four miRNAs were present in some holometabolous (miR-125-5p, miR-2765, miR-375, miR-92b-3p), a particular case, miR-2765 was present in the orders Coleoptera, Lepidoptera, and Hymenoptera but not Diptera; five miRNAs were shared between holometabolous and the order Hemiptera (miR-100, miR-184, miR-7, miR-965, miR-iab-4-5p). Finally, the miRNA conserved in all holometabolous and hemimetabolous orders was miR-10.

miRNAs biological function in Ae. aegypti. We found 55 miRNAs from Ae. aegypti using miRDeep2 (biological database) tool (Supplementary Material Table S1). Figure 5A shows that these 55 were distributed in 8 different biological function categories, which in percentage were as follows: embryo development (ED) 23.6\%, basal functions (BF) 21.8\%, metamorphosis (Met) $21.8 \%$, sexuality (Sex) 12.7\%, unknown (Unk) 9.09\%, immune response (IR) $5.45 \%$, blood digestion (BD) $3.64 \%$ and detoxification (Detox) $1.82 \%$.

There were 4 categorization clusters (Fig. 5B): Culicidae with high DE proportion, Diptera with BF, holometabolous (Ho) with BF and holometabolous + hemimetabolous ( $\mathrm{Ho}+\mathrm{He}$ ) with Met. Three parameters had expression levels (upregulated, basal and downregulated expression) and life stages (eggs, females and males) (Fig. 5C), where eggs showed DD upregulation and BD, BF, IR and Met downregulation; females showed BD, BF, Detox and Sex upregulated. Finally, males exhibited BD downregulated. 


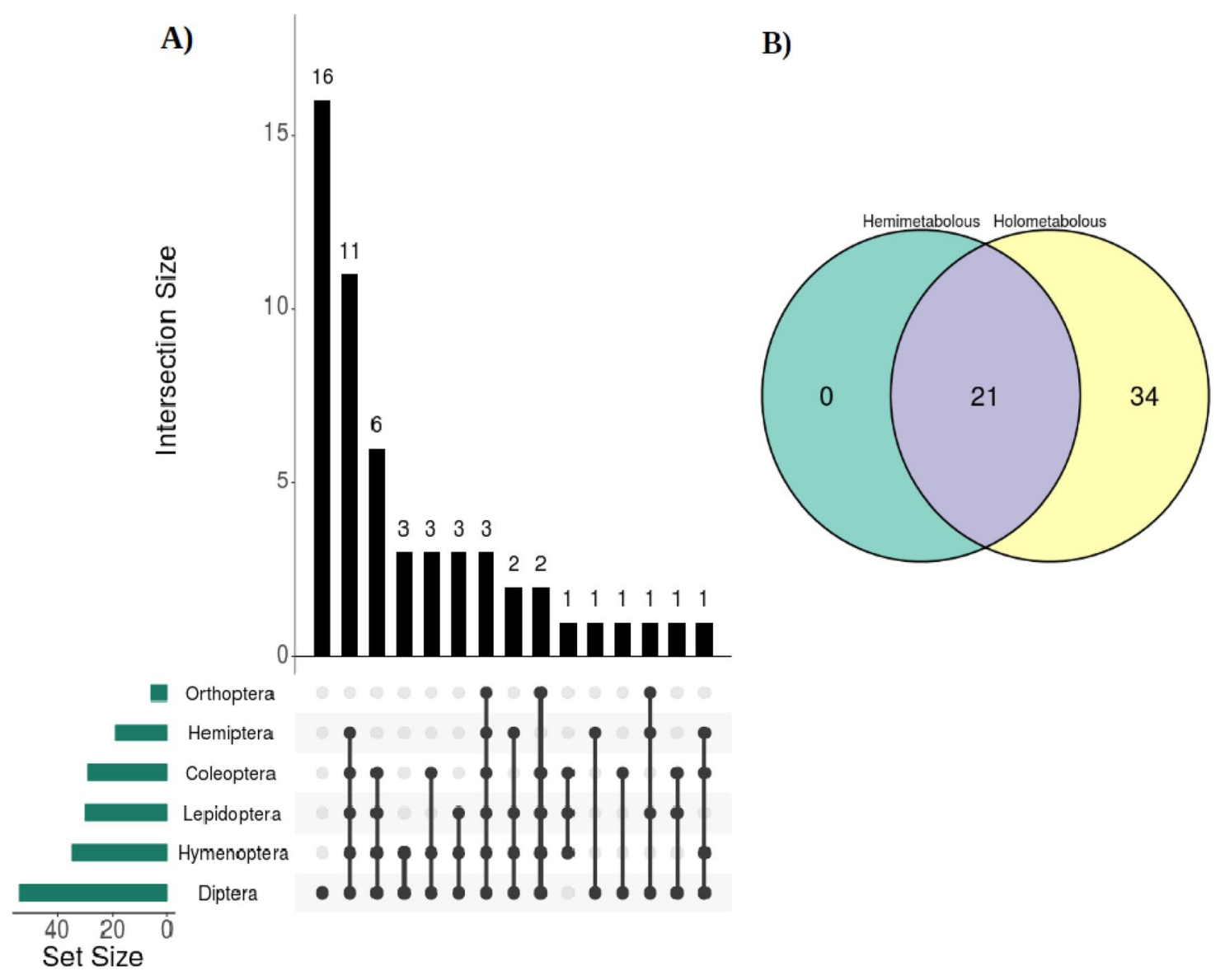

Figure 2. An interaction graph of the 55 conserved miRNAs from Ae. aegypti in the different orders analyzed. (A) In the UpSet diagram, on the x-axis, a 15 different conservation pattern matrix with miRNAs representing the 6 orders (dotted black lines) is presented; likewise, miRNA abundances of each pattern are illustrated on the y-axis in black bar graph form. (B) Distribution Venn diagram of the 55 miRNAs between the two metamorphoses types analyzed.

\section{Discussion}

Metamorphosis diversity in insects (ametabolous, hemimetabolous and holometabolous) is a fundamental part of their evolution, being a determining factor in the conservation of these organisms ${ }^{15}$. So far, different miRNAs, such as miR-let-7, miR-100 and miR-125, have been conserved and participate in metamorphosis in different organisms such as Blattella germanica Linnaeus, 1767 (hemimetabolous) ${ }^{16}$ and Anopheles gambiae (holometabolous $)^{17-19}$. We showed that 34 miRNAs corresponded to only one or more holometabolous organism, classified in the orders Diptera, Hymenoptera, Lepidoptera and Coleoptera and that 21 miRNAs shared conservation with some hemimetabolous insects.

We observed an abundance of miRNAs, i.e., 12, related to the metamorphosis process (Fig. 5A, yellow segment, and Supplementary Table S1); these miRNAs have been associated with different metamorphosis functions in other insects, such as bantam- $5 \mathrm{p}^{11}$, let-7, miR-252-5 $\mathrm{p}^{20}$ and miR-315-5 $\mathrm{p}^{21}$, regulating wing development. miR$263 \mathrm{a}-5 \mathrm{p}^{22}$ and miR-282-5 $\mathrm{p}^{23}$ have been found in neuronal regulation signals. miR-1890 regulates JHA15 juvenile hormone expression in Ae. aegypti ${ }^{5}$; miR-133 is upregulated in Drosophila melanogaster pupation ${ }^{24}$. Of the 12 miRNAs related to the metamorphosis process, eight (let-7, miR-14, miR-263a-5p, miR-276-3p, miR-276-5p, miR-315-5p and miR-965) have been found to be conserved in holometabolous as well as hemimetabolous organisms (Fig. 5B, Ho $+\mathrm{He}$ ). Some of these miRNAs have been reported to be in great abundance in insects ${ }^{25-27}$ and have been associated with wing development in hemimetabolous metamorphosis in B. germanica ${ }^{28-30}$. This may suggest that the development of wings has been regulated at the miRNomic level by organisms conserved along the evolution of the Insecta.

Another portion contains 13 miRNAs (Fig. 5A, purple section; Supplementary Table S1); six of these showed overexpression in eggs [Fig. 5C, second bar "Eggs (Up)", (Supplementary Table S1)], but they are not related to specific biological events as yet. We observed miR-10 and miR-71-5p overexpression in holometabolous and hemimetabolous insects. Only five have been described previously in D. melanogaster embryos playing developmental functions: miR-307 regulated cell death processes through the silencing of wntless $^{6}$; miR-33 in cell proliferation events by silencing the genes $C D K 6$ y $C C N D 1^{31}$; miR-9a in muscle myofibril development by silencing troponin- $\mathrm{T}^{32}$; and miR-9c-5p in the cleaning of unstable maternal transcripts ${ }^{33}$. Finally, miR-iab-4-5p 


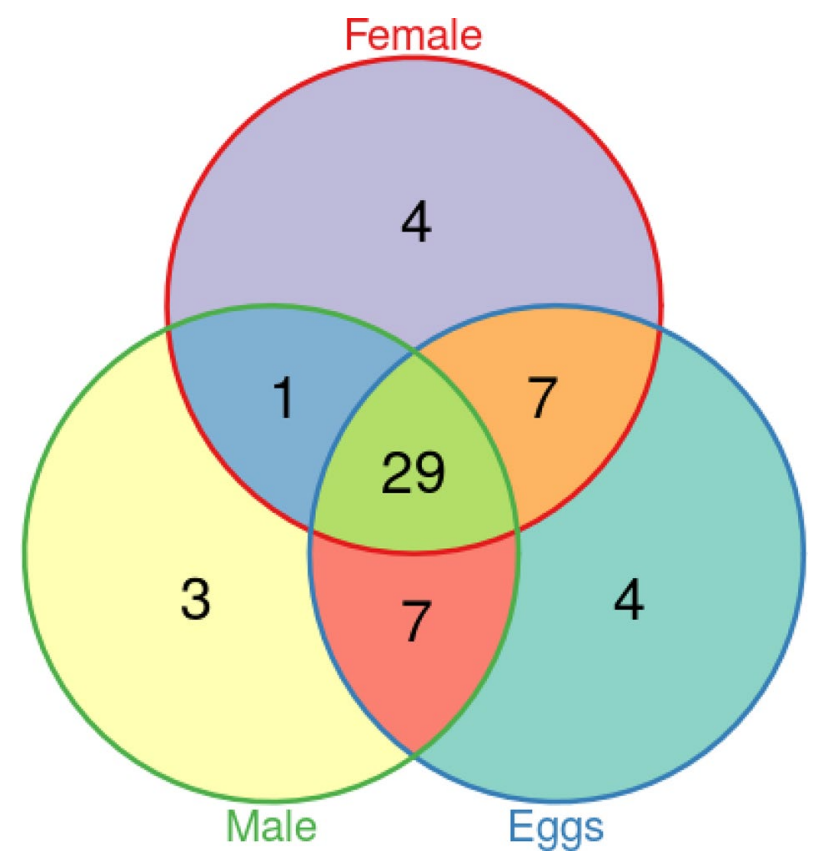

Figure 3. Venn diagram with the distribution of miRNAs conserved in organisms of the class Insecta among the three life stages analyzed.

A)

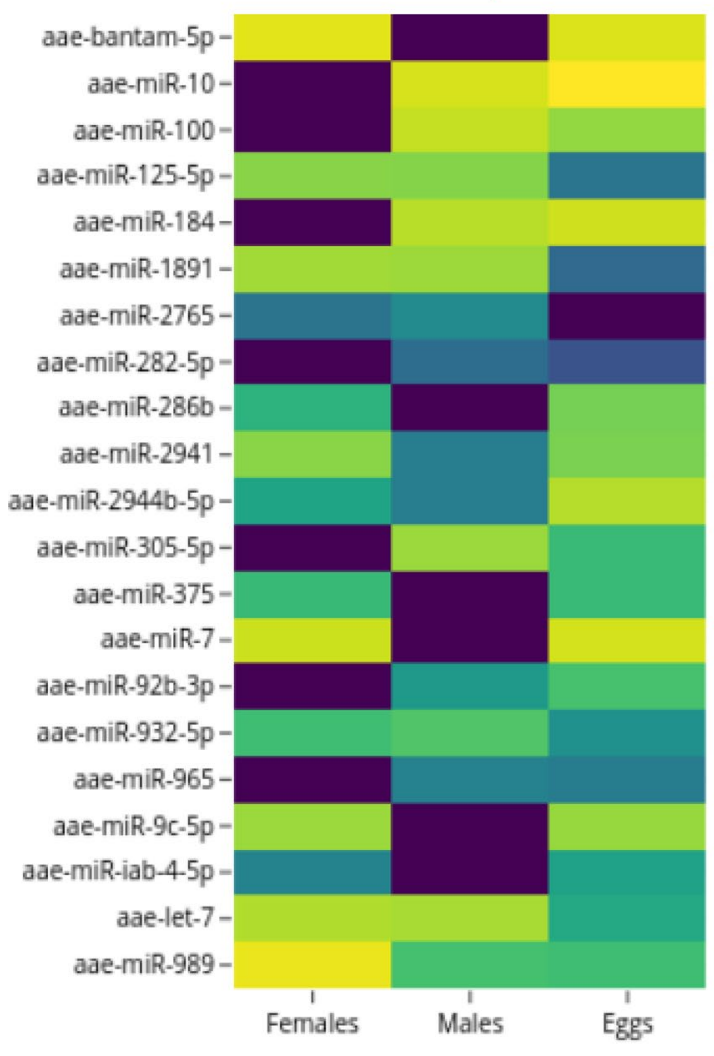

B)

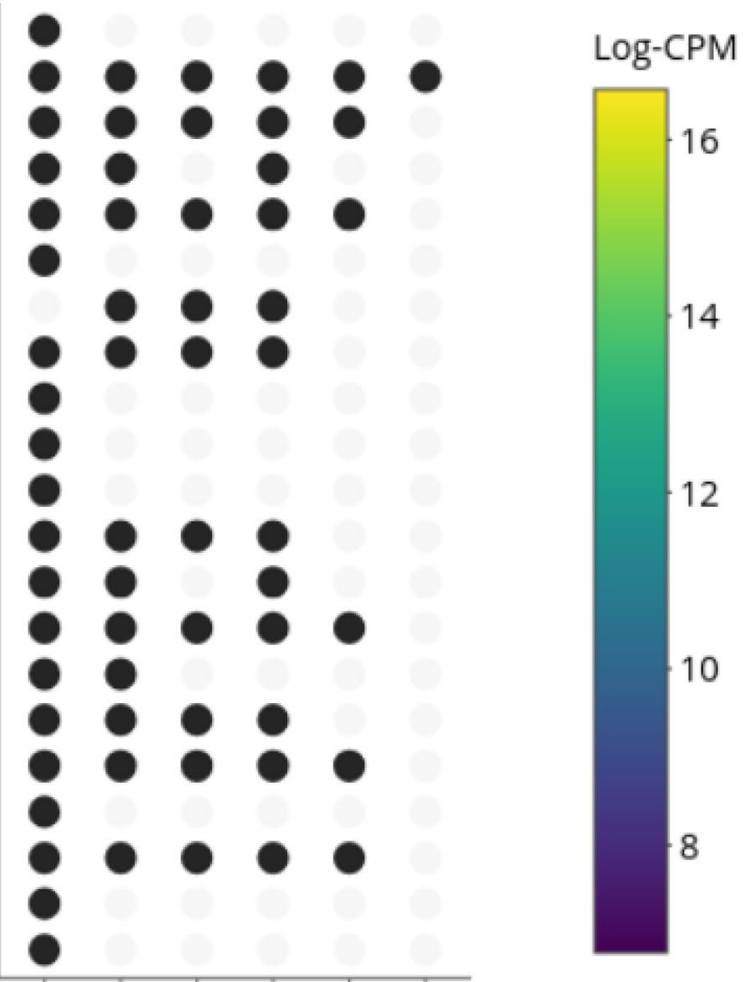

Figure 4. Differential expression of 21 miRNAs in female, male and eggs from Ae. aegypti and their distribution in the class Insecta. (A) Heatmap of the 21 miRNAs with DE (y-axis) in 3 approaches analyzed (x-axis) and (B) conservation patterns of each miRNA between insect orders. 
A)

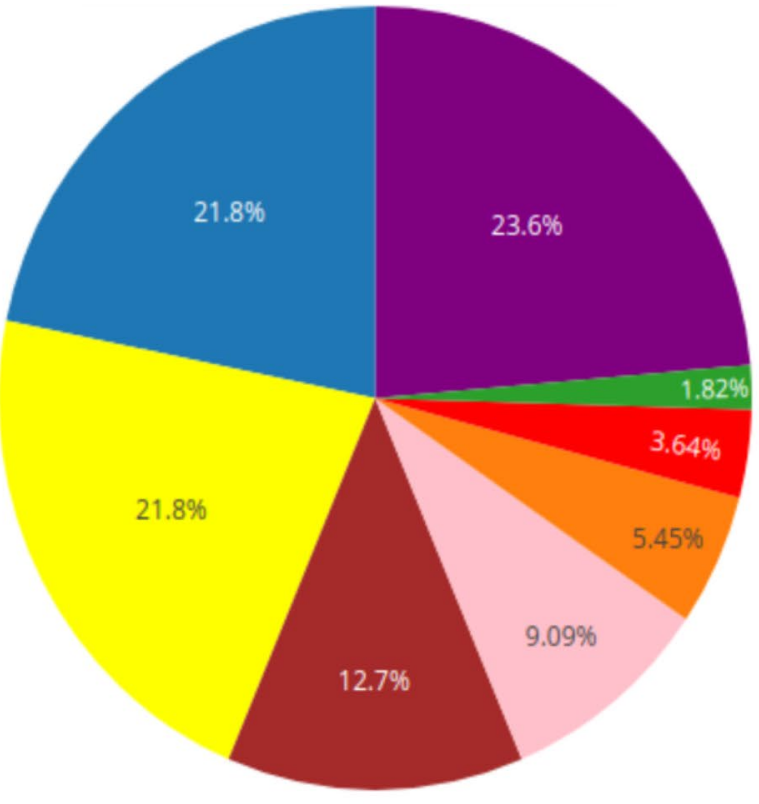

Embryo development

Basal functions

Metamorphosis

Sexuality

Unknown

Immune response

Blood digestion

Detoxification
B)

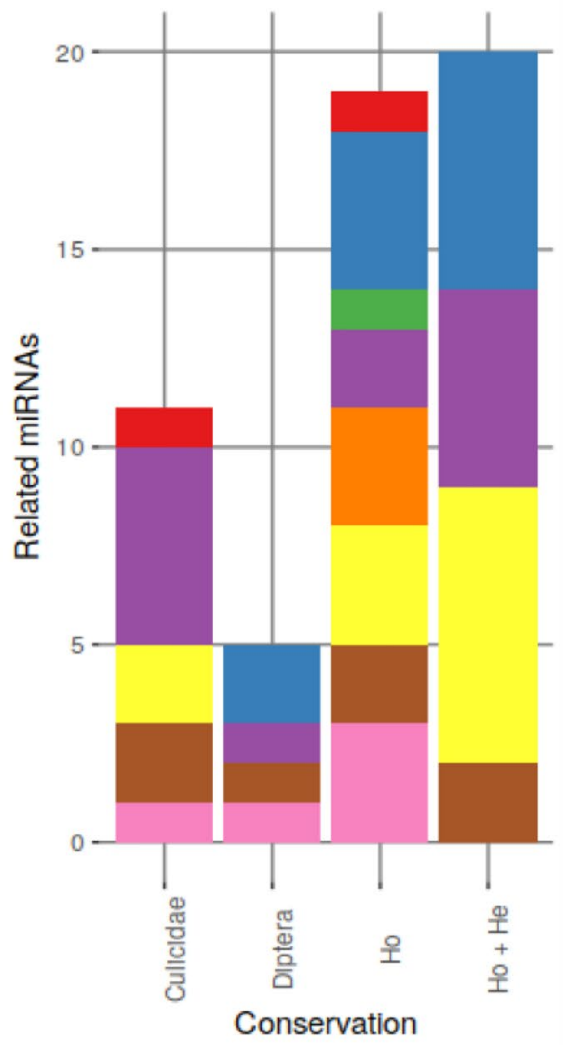

C)

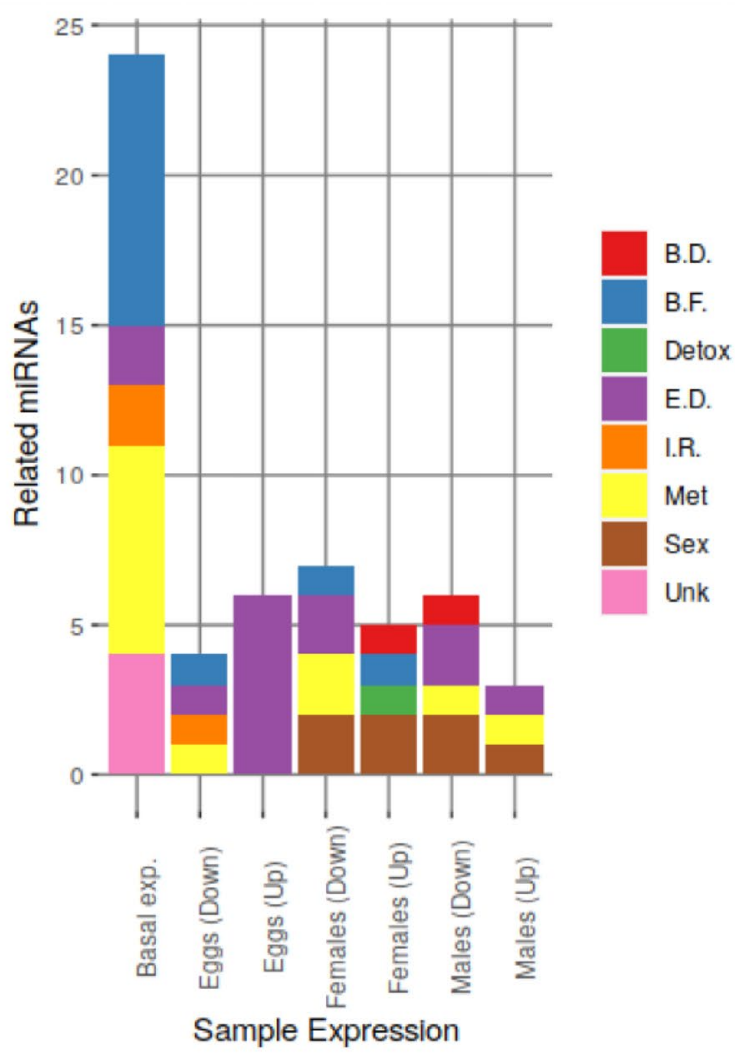

Figure 5. Biological function correlation with abundance of the 55 miRNAs in Ae. aegypti. (A) miRNAs distribution correlated with biological function, (B) miRNA distribution based on categorization clusters and (C) miRNAs expression levels in three life stages. Ho holometabolous, He hemimetabolous.

regulating the distribution and development of the wings and halters through the silencing of Ultrabithorax ${ }^{34}$. Thus, we can suggest embryonic development function for these 5 miRNAs in Ae. aegypti.

Five miRNAs (miR-1891, miR-286b, miR-2944a-5p, miR-2944b-5p and miR-996) were found conserved only in the members of the family Culicidae (Fig. 5B, bar 1 "Culicidae", Supplementary Table S1); only two 
(miR-1891 and miR-996) have been reported overexpressed in eggs of Ae. aegypti, Anopheles gambiae and An. stephensi Liston $1901^{35,36}$, and three (miR-286b, miR-2944a-5p, miR-2944b-5p) were highly expressed in the early development stages of mosquitoes ${ }^{35,37}$. The family formed by miR-996, miR-279, and miR-296 have undergone some duplication events along insect evolution, making it diversify in holometabolous organisms ${ }^{38}$. Thus, we suggested that miRNAs conserved specifically in Culicidae could have undergone similar events that would have led to miRNomic regulation in embryonic stages in a specific way.

Discrepancies were found between previous studies and our findings regarding the following miRNAs: miR9a has been reported in embryo development function in D. melanogaster eggs ${ }^{32}$, but in contrast, no significant changes were found in the biological approaches that we used (eggs, females and males); miR-71-5p with overexpression in Manduca sexta (Linnaeus 1763) eggs $^{39}$ was in contrast to the expression in males in our findings; miR-10 was overexpressed in D. melanogaster, T. castaneum, Apis mellifera (Linnaeus 1758) and B. germanica eggs $^{40,41}$ and downregulated in females according to our results. Thus, as explained above, we can infer new and different roles for these miRNAs in Ae. aegypti.

Twelve miRNAs conserved between Ae. aegypti and the class Insecta were categorized in diverse basal functions such as neuronal signaling, hypoxia functions, nervous system, and circadian cycle (Fig. 5A, blue section; Supplementary Table S1). The functions of these miRNAs are: miR-1, regulating the architecture and maintenance of D. melanogaster cardiac tissue through DELTA silencing ${ }^{21}$; miR-137, in neuronal signaling of Drosophila Parkinson disease models ${ }^{42}$; miR-190, orchestrating the functions related to hypoxia in D. melanogaster ${ }^{43}$; miR263b-5p and miR-279, in the circadian cycle in D. melanogaster ${ }^{44}$; miR-2c and miR-31, in the maintenance of the nervous system of $D$. melanogaster ${ }^{45,46}$ and miR-957, in the interaction Cx. quinquefasciatus/Western Nile virus $(\mathrm{WNV})^{47}$; miR-9b, silencing serine/threonine kinase protein in Drosophila ${ }^{48}$; miR-932-5p, in the neuroplasticity mechanisms and long-term memory of $A$. mellifera ${ }^{49}$; miR-305-5p, in the intestinal homeostasis of $D$. melanogaster ${ }^{50}$; and miR-8-5p, in post-embryonic development ${ }^{51}$ and growth factor regulation of body fat in larval stages ${ }^{40}$ in D. melanogaster.

Of these 12 miRNAs, 9 showed a basal expression in the life stages of Ae. aegypti analyzed in the present study (Fig. 5C, bar 1 "Basal exp."; Supplementary Table S1) and the remaining 3, were downregulated in eggs (miR932-5p) or upregulated (miR-31) and downregulated (miR-305-5p) in females. Conservation profiles of the 12 miRNAs showed 4 with conservation only in holometabolous, 6 in holometabolous and hemimetabolous and 2 specifically in Diptera (Fig. 5B). Of the basal function of the miRNAs with holometabolous and hemimetabolous conservation, miR-2c and miR-8-5p have different biological functions depending on the type of metamorphosis that was explored. For the hemimetabolous functions for these miRNAs, miR-2c was related to metamorphosis processes through the silencing of KR-H1 in B. germanica ${ }^{28}$ and miR-8-5p in chitin biosynthesis in Locusta migratoria Linnaeus $1758^{52}$ and motor coordination of B. germanica ${ }^{53}$.

Other biological functions were observed (sex, blood digestion and detoxification) that despite having a low abundance are relevant in the biology of Ae. aegypti. miRNAs were found to be related to sexual development, where 2 were found to be unique for Culicidae (miR-2942 and miR-989) (Fig. 5B, bar 1 "Culicidae"; Supplementary Table S1), which regulates ovary development in Ae. aegypti, Cx. quinquefasciatus and An. stephensi $i^{14,54-56 .}$

On the other hand, miR-193 was shown to be conserved in the order Diptera (Fig. 5B, bar 2 "Diptera"; Supplementary Table S1), being overexpressed in D. melanogaster ${ }^{57}$. Also, the experimental design did not consider sex as a factor for expression analysis, so our results pointed to be a possible function in feminization. The battery of miR-7 and miR-993 was conserved in the holometabolous organisms (Fig. 5B, bar 3 "Ho"; Supplementary Table S1), where it has been observed that they fulfill masculinization roles in D. melanogaster ${ }^{58,59}$. Another battery of miR-184 and miR-100 was conserved in holo- and hemimetabolous insects (Fig. 5B, bar 4 "Ho + He"; Supplementary Table S1), both being highly expressed in males of An. anthropophagus Xu \& Feng $1975^{60}$. miR184 have been associated with regulation of ovary development in D. melanogaster ${ }^{21}$ and with high expression in females of the $6^{\text {th }}$ nymphal stage in B. germanica ${ }^{30}$ and miR-100 with essential metamorphosis regulation conserved among holometabolous and hemimetabolous insects ${ }^{28}$. Our results suggest that the feminization of mosquitoes may require a miRNomic regulation conserved uniquely in Culicidae.

Two miRNAs were found associated with blood digestion (Fig. 5A, red section); the miR-1175-5p was strictly conserved in Culicidae (Fig. 5B, bar 1 "Culicidae"; Supplementary Table S1) being in high concentrations in females of An. stephensi, An. gambiae, and Cx. quinquefasciatus ${ }^{14,36}$, and miR-375 regulating the silencing of immunological cactus genes and REL1 in Ae. aegypti ${ }^{7}$.

Finally, only miR-285 was found to be associated with detoxification functions. Previously, it has been described in a resistant strain $(91-\mathrm{R})$ of $D$. melanogaster in exposure to $\mathrm{DDT}^{61}$. This agrees with our results of overexpression in females because resistant strains were not analyzed in this study, and we used the susceptible reference strain New Orleans. However, expression findings infer that miR-285 plays an essential role in the development of insecticide resistance in a specific way in Diptera.

In conclusion, the sequencing of miRNAs in eggs and adults (males and females) of Ae. aegypti showed a total of 55 miRNAs with relevant conservation with other members of the class Insecta. Of these miRNAs, 32 showed DE in the stages analyzed, showing to be related to development, metamorphosis, and sex functions, among others. On the other hand, 12 miRNAs with basal expressions were shown to be mainly related to diverse biological functions. While a lower abundance of conservation was expected in specific functions, such as detoxification, blood digestion, and immune response, there was a predominance of highly conserved miRNAs related to embryonic development and metamorphosis processes or a diversity of conservation clusters in miRNAs related to sexuality. These results suggest that the development of wings, with miR-iab-4-5p and masculinization, with the battery of miR-7 and miR-993, could be ancestral miRNomic mechanisms.

We propose to coin the word "phylorthology" to refer to the phylogenetic relationship that species have with relation in a conserved sequence in $100 \%$. 


\section{Methods}

General strategy. The general strategy consisted of obtaining miRNA sequences from 3 life stages in Ae. aegypti (males, females, and eggs). Total RNA was extracted, and the corresponding miRNA fractions were sequenced. These sequences were annotated and subjected to conservation analysis against the miRNAs available in the class Insecta. Those with significant conservation (E value $\leq 0.005)$ were subjected to interaction graphics and cladograms, to observe their abundance and diverse patterns of conservation between the organisms of the class Insecta. Finally, a DE analysis of these miRNAs was evaluated using a likelihood ratio statistical analysis.

Sample collection. Ae. aegypti specimens belonging to the New Orleans strain were bred at the insectarium of the Medical Entomology Laboratory of the Facultad de Ciencias Biologicas in the Universidad Autonoma de Nuevo Leon. Breeding of the mosquitoes was performed under laboratory conditions with a temperature of $28 \pm 2{ }^{\circ} \mathrm{C}$, a light/dark photoperiod of $12 / 12 \mathrm{~h}$ and relative humidity of $70 \pm 2 \%$. A total of 3000 eggs, 100 males and 100 females were separated, and miRNA extraction from the specimens was carried out with the commercial kit miRNeasy Mini, following the provider instructions (Qiagen, Germantown, MD, USA). RNA extracted was treated with RQ1 RNase-Free Dnase (Promega, San Luis Obispo, CA, USA) to remove genomic DNA traces. Standard spectrophotometry (Thermo Fisher, Waltham, MA, USA) and agarose gel electrophoresis were used to evaluate RNA purity and integrity, respectively.

Next generation sequencing. RNA of acceptable quality and quantity was sent to BGI Global Genomics Services (Yantian District, Shenzhen, China) for sequencing of the small fraction (less than 30 base pairs) with Illumina Solexa technology and ligation of pair adaptors to their $5^{\prime}$ and $3^{\prime}$ ends.

miRNA annotation. miRNA annotations were made with the tool miRDeep $2^{62}$, using as a reference genome the assembled version of Ae. aegypti (AaegL5), and as a precursor reference the available structures of miRNAs in miRBase v $22^{63}$ of the 30 organisms of the subphylum Hexapoda. The analysis was run with a minimum cut-off of $18 \mathrm{nt}$, and only the miRNAs that showed a miRDeep score $\geq 4$ were considered, on the basis of those described by Friedländer et al. ${ }^{62}$ and our own criteria.

Phylogenetic and conservation analysis. The annotated miRNAs that had phylogenetic conservations (phyloortology) with organisms of class Insecta were identified through local alignments against the database of miRNAs available for Hexapoda with the BLASTN tool of miRBase. Mature and precursor sequences with E values $\leq 0.005$ were considered conserved and those with values $>0.005$ were discarded. Subsequently, the abundance of miRNAs conserved with our results were quantified and categorized by insect species. A cladogram was generated with taxonomic data ${ }^{64}$ of every species with the tools of the software Rv3.6.0 $0^{65}$ : ggtree1.14.6 $6^{66}$, treeio1.6. $2^{67}$, devtools2 $.0 .2^{68}$, phytools $0.6-72^{69}$ and ggimage $0.2 .1^{70}$. miRNA presence in the different insect orders and metamorphosis types (holometabolous and hemimetabolous) were observed. These trend analyses were made through visualizations of interaction of UpSet and Venn diagrams through the use of the graphic interactive tool intervene ${ }^{71}$ and the package UpSetRv1.3.3.

Differential expression analysis. For the expression analyses, only miRNAs that showed conservation were considered; the total reads were obtained by the mean of each of the approaches explored. A Venn diagram was made to show the dispersion of these miRNAs between the 3 different biological approaches (eggs, females and males) and to discard the miRNAs with specific expression in only one explored biological approach. The mean of the readings was put through depuration and normalization protocols according to Law et al. ${ }^{73}$, where data expression of the readings was transformed into counts per million (CPM). This value was expressed in log2 $(\log 2-\mathrm{CPM})$ and was normalized using Trimmed Mean of M values (TMM) method. For the determination of the miRNAs with DE in the three contrasted approaches (eggs vs females, eggs vs males and, females vs males), a generalized linear model and a likelihood ratio likelihood (LRT) were used ${ }^{74}$. The miRNAs with a DE were considered if they showed LRT values of $>4$ and a $\mathrm{p}$ value $\leq 0.01$; their expression values $(\log 2-\mathrm{CPM})$ were used for the plotting of graphs and heat maps through the $\mathrm{R}$ tools of graphics development heatmaply ${ }^{75}, \mathrm{Glimma}^{76}$ and RColorBrewer ${ }^{77}$. The R tools limma $3.40 .0^{78}$ and edgeR $3.24 .3^{79}$ were used for statistical analyses.

Received: 14 September 2020; Accepted: 5 April 2021

Published online: 21 May 2021

\section{References}

1. Clements, A. N. The Biology of Mosquitoes. Development, Nutrition and Reproduction 509 (Chapman \& Hall, 1992).

2. Dávalos, B. E. et al. Urban and semi-urban mosquitoes of Mexico City: A risk for endemic mosquito-borne disease transmission. PLoS ONE 14, e0212987. https://doi.org/10.1371/journal.pone.0212987 (2019).

3. Vilcinskas, A. Insect Biotechnology 268 (Springer, 2011). https://doi.org/10.1007/978-90-481-9641-8.

4. Kehl, T. et al. About miRNAs, miRNA seeds, target genes and target pathways. Oncotarget 8, 107167-107175. https://doi.org/10. 18632/oncotarget.22363 (2017).

5. Lucas, K. J., Zhao, B., Roy, S., Gervaise, A. L. \& Raikhel, A. S. Mosquito-specific microRNA-1890 targets the juvenile hormoneregulated serine protease JHA15 in the female mosquito gut. RNA Biol. 12, 1383-1390. https://doi.org/10.1080/154762862015. 1101525 (2015).

6. Zhang, P., Zhou, L., Pei, C., Lin, X. \& Yuan, Z. Dysfunction of Wntless triggers the retrograde Golgi-to-ER transport of Wingless and induces ER stress. Sci. Rep. 6, 19418. https://doi.org/10.1038/srep19418 (2016). 
7. Hussain, M., Walker, T., O’Neill, S. L. \& Asgari, S. Blood meal induced microRNA regulates development and immune associated genes in the Dengue mosquito vector, Aedes aegypti. Insect Biochem. Mol. 43, 146-152. https://doi.org/10.1016/j.ibmb.2012.11. $005(2013)$

8. Osei-Amo, S., Hussain, M., O’Neill, S. L. \& Asgari, S. Wolbachia-induced aae-miR-12 miRNA negatively regulates the expression of MCT1 and MCM6 genes in Wolbachia-infected mosquito cell line. PLoS ONE 7, e50049. https://doi.org/10.1371/journal.pone/ 0050049 (2012).

9. Zhang, G., Hussain, M., O'Neill, S. L. \& Asgari, S. Wolbachia uses a host microRNA to regulate transcripts of a methyltransferase, contributing to dengue virus inhibition in Aedes aegypti. Proc. Natl. Acad. Sci. 110, 10276-10281. https://doi.org/10.1073/pnas. 1303603110 (2013).

10. Liu, S., Lucas, K. J., Roy, S., Ha, J. \& Raikhel, A. S. Mosquito-specific microRNA-1174 targets serine hydroxymethyltransferase to control key functions in the gut. Proc. Natl. Acad. Sci. 111, 14460-14465. https://doi.org/10.1073/pnas.1416278111 (2014).

11. Bryant, B., Macdonald, W. \& Raikhel, A. S. microRNA miR-275 is indispensable for blood digestion and egg development in the mosquito Aedes aegypti. Proc. Natl. Acad. Sci. 107, 22391-22398. https://doi.org/10.1073/pnas.1016230107 (2010).

12. Marco, A., Hui, J. H. L., Ronshaugen, M. \& Griffiths-Jones, S. Functional shifts in insect microRNA evolution. Genome Biol. Evol. 2, 686-696. https://doi.org/10.1093/gbe/evq053 (2010).

13. Hong, S. et al. Identification of differentially expressed microRNAs in Culex pipiens and their potential roles in pyrethroid resistance. Insect Biochem Mol. 55, 39-50. https://doi.org/10.1016/j.ibmb.2014.10.007 (2014).

14. Skalsky, R. L., Vanlandingham, D. L., Scholle, F., Higgs, S. \& Cullen, B. R. Identification of microRNAs expressed in two mosquito vectors, Aedes albopictus and Culex quinquefasciatus. BMC Genom. 11, 119. https://doi.org/10.1186/1471-2164-11-119 (2010).

15. Truman, J. W. \& Riddiford, L. M. The origins of insect metamorphosis. Nature 401, 447-452. https://doi.org/10.1038/46737 (1999).

16. Rubio, M., de Horna, A. \& Belles, X. MicroRNAs in metamorphic and non-metamorphic transitions in hemimetabolan insect metamorphosis. BMC Genom. 13, 386. https://doi.org/10.1186/1471-2164-13-386 (2012).

17. Jain, S., Rana, V., Tridibes, A., Sunil, S. \& Bhatnagar, R. K. Dynamic expression of miRNAs across immature and adult stages of the malaria mosquito Anopheles stephensi. Parasit. Vectors 8, 179. https://doi.org/10.1186/s13071-015-0772-y (2015).

18. Jain, S., Shrinet, J., Tridibes, A., Bhatnagar, R. \& Sunil, S. miRNA-mRNA conflux regulating immunity and oxidative stress pathways in the midgut of blood-fed Anopheles stephensi. Non-Coding RNA 1, 222-245. https://doi.org/10.3390/ncrna1030222 (2015).

19. Roush, S. \& Slack, F. J. The let-7 family of microRNAs. Trends Cell Biol. 18, 505-516. https://doi.org/10.1016/j.tcb.2008.07.007 (2008).

20. Towler, B. P. et al. The 3'-5' exoribonuclease Dis3 regulates the expression of specific microRNAs in Drosophila wing imaginal discs. RNA Biol. 12, 728-741. https://doi.org/10.1080/15476286.2015.1040978 (2015).

21. Jones, C. I. \& Newbury, S. F. Functions of microRNAs in Drosophila development. Biochem. Soc. Trans. 38, 1137-1143. https:// doi.org/10.1042/BST0381137 (2010).

22. Hilgers, V., Bushati, N. \& Cohen, S. M. Drosophila microRNAs 263a/b Confer robustness during development by protecting nascent sense organs from apoptosis. PLoS Biol. 8, e1000396. https://doi.org/10.1371/journal.pbio.1000396 (2010).

23. Wu, P., Jiang, X., Guo, X., Li, L. \& Chen, T. Genome-wide analysis of differentially expressed microRNA in Bombyx mori infected with nucleopolyhedrosis virus. PLoS ONE 11, e0165865. https://doi.org/10.1371/journal.pone.0165865 (2016)

24. Sempere, L. F., Sokol, N. S., Dubrovsky, E. B., Berger, E. M. \& Ambros, V. Temporal regulation of microRNA expression in Drosophila melanogaster mediated by hormonal signals and Broad-Complex gene activity. Dev. Biol. 259, 9-18. https://doi.org/10.1016/ S0012-1606(03)00208-2 (2003).

25. Wu, W. et al. Characterization and comparative profiling of MicroRNAs in a sexual dimorphism insect, Eupolyphaga sinensis Walker.. PLoS ONE 8(4), e59016. https://doi.org/10.1371/journal.pone.0059016 (2013).

26. Legeai, F. et al. Bioinformatic prediction, deep sequencing of microRNAs and expression analysis during phenotypic plasticity in the pea aphid, Acyrthosiphon pisum. BMC Genom. 11, 281. https://doi.org/10.1186/1471-2164-11-281 (2010).

27. Wei, Y., Chen, S., Yang, P., Ma, Z. \& Kang, L. Characterization and comparative profiling of the small RNA transcriptomes in two phases of locust. Genome Biol. 10, R6. https://doi.org/10.1186/gb-2009-10-1-r6 (2009).

28. Belles, X. MicroRNAs and the evolution of insect metamorphosis. Annu. Rev. Entomol. 62, 111-125. https://doi.org/10.1146/annur ev-ento-031616-034925 (2017).

29. Li, X. et al. Comparative profiling of microRNAs in the winged and wingless English grain aphid, Sitobion avenae (F) (Homoptera: Aphididae). Sci. Rep. 6, 35668. https://doi.org/10.1038/srep35668 (2016).

30. Cristino, A. S., Tanaka, E. D., Rubio, M., Piulachs, M. D. \& Belles, X. Deep sequencing of organ- and stage-specific microRNAs in the evolutionarily basal insect Blattella germanica.(L) (Dictyoptera, Blattellidae). PLoS ONE 6, 19350. https://doi.org/10.1371/ journal.pone.0019350 (2011).

31. Iwakiri, Y. A role of miR-33 for cell cycle progression and cell proliferation. Cell Cycle 11, 1057-1057. https://doi.org/10.4161/cc. 11.6.19744 (2012).

32. Katti, P., Thimmaya, D., Madan, A. \& Nongthomba, U. Overexpression of miRNA-9 generates muscle hypercontraction through translational repression of troponin-T in Drosophila melanogaster indirect flight muscles. G3 Genes Genom Genet 7, 3521-3531. https://doi.org/10.1534/g3.117.300232 (2017).

33. Marco, A. Selection against maternal microRNA target sites in maternal transcripts. G3 Genes Genom Genet. 5, 2199-2207. https:// doi.org/10.1534/g3.115.019497 (2015).

34. Ronshaugen, M., Biemar, F., Piel, J., Levine, M. \& Lai, E. C. The Drosophila microRNA iab-4 causes a dominant homeotic transformation of halteres to wings. Genes Dev. 19, 2947-2952. https://doi.org/10.1101/gad.1372505 (2005)

35. Akbari, O. S. et al. The developmental transcriptome of the mosquito Aedes aegypti, an invasive species and major arbovirus vector. G3 Genes Genom. Genet. 3, 1493-1509. https://doi.org/10.1534/g3/113.006742 (2013).

36. Li, S., Mead, E. A., Liang, S. \& Tu, Z. Direct sequencing and expression analysis of a large number of miRNAs in Aedes aegypti and a multi-species survey of novel mosquito miRNAs. BMC Genom. 10, 581. https://doi.org/10.1186/1471-2164-10-581 (2009).

37. Castellano, L. et al. The germline of the malaria mosquito produces abundant miRNAs, endo-siRNAs, piRNAs and 29-nt small RNAs. BMC Genom. 16, 100. https://doi.org/10.1186/s12864-015-1257-2 (2015).

38. Mohammed, J., Siepel, A. \& Lai, E. C. Diverse modes of evolutionary emergence and flux of conserved microRNA clusters. RNA 20, 1850-1863. https://doi.org/10.1261/rna.046805.114 (2014).

39. Zhang, X. et al. Identification and developmental profiling of conserved and novel microRNAs in Manduca sexta. Insect Biochem. Mol. 42, 381-395. https://doi.org/10.1016/j.ibmb.2012.01.006 (2012).

40. Ylla, G., Piulachs, M. D. \& Belles, X. Comparative analysis of miRNA expression during the development of insects of different metamorphosis modes and germ-band types. BMC Genom. 18, 774. https://doi.org/10.1186/s12864-017-4177-5 (2017).

41. Zondag, L., Dearden, P. K. \& Wilson, M. J. Deep sequencing and expression of microRNAs from early honeybee (Apis mellifera) embryos reveals a role in regulating early embryonic patterning. BMC Evol. Biol. 12, 211. https://doi.org/10.1186/1471-2148-12211 (2012).

42. Kong, Y. et al. High throughput sequencing identifies microRNAs Mediating $\alpha$-synuclein toxicity by targeting neuroactive-ligand receptor interaction pathway in early stage of Drosophila Parkinson's disease model. PLoS ONE 10, e0137432. https://doi.org/10. 1371/journal.pone.0137432 (2015).

43. De Lella Ezcurra, A. L. et al. miR-190 enhances HIF-dependent responses to hypoxia in Drosophila by inhibiting the prolyl4-hydroxylase fatiga. PLoS Genet. 12, e1006073. https://doi.org/10.1371/journal.pgen.1006073 (2016). 
44. Xue, Y. \& Zhang, Y. Emerging roles for microRNA in the regulation of Drosophila circadian clock. BMC Neurosci. 19, 1. https:// doi.org/10.1186/s12868-018-0401-8 (2018).

45. Foo, L. C. Cyclin-dependent kinase 9 is required for the survival of adult Drosophila melanogaster glia. Sci. Rep. 7, 6796. https:// doi.org/10.1038/s41598-017-07179-8 (2017).

46. Marco, A., Hooks, K. \& Griffiths-Jones, S. Evolution and function of the extended miR-2 microRNA family. RNA Biol. 9, 242-248. https://doi.org/10.4161/rna.19160 (2012).

47. Asgari, S. Role of microRNAs in insect host-microorganism interactions. Front. Physiol. 2, 48. https://doi.org/10.3389/fphys.2011. 00048 (2011).

48. Mead, E. A. \& Tu, Z. Cloning, characterization, and expression of microRNAs from the Asian malaria mosquito, Anopheles stephensi. BMC Genom. 9, 244. https://doi.org/10.1186/1471-2164-9-244 (2008).

49. Cristino, A. S. et al. Neuroligin-associated microRNA-932 targets actin and regulates memory in the honeybee. Nat. Commun. 5, 5529. https://doi.org/10.1038/ncomms6529 (2014).

50. Foronda, D., Weng, R., Verma, P., Chen, Y. W. \& Cohen, S. M. Coordination of insulin and Notch pathway activities by microRNA miR-305 mediates adaptive homeostasis in the intestinal stem cells of the Drosophila gut. Genes Dev. 28, 2421-2431. https://doi. org/10.1101/gad.241588.114 (2014).

51. Ninova, M., Ronshaugen, M. \& Griffiths-Jones, S. Conserved temporal patterns of microRNA expression in Drosophila support a developmental hourglass model. Genome Biol. Evol. 6, 2459-2467. https://doi.org/10.1093/gbe/evu182 (2014).

52. Yang, M. et al. miR-71 and miR-263 jointly regulate target genes chitin synthase and chitinase to control locust molting. PLoS Genet. 12, e1006257. https://doi.org/10.1371/journal.pgen.1006257 (2016).

53. Rubio, M., Montañez, R., Perez, L., Milan, M. \& Belles, X. Regulation of atrophin by both strands of the mir-8 precursor. Insect Biochem. Mol. 43, 1009-1014. https://doi.org/10.1016/j.ibmb.2013.08.003 (2013).

54. Feng, X., Wu, J., Zhou, S., Wang, J. \& Hu, W. Characterization and potential role of microRNA in the Chinese dominant malaria mosquito Anopheles sinensis (Diptera: Culicidae) throughout four different life stages. Cell Biosci. 8, 29. https://doi.org/10.1186/ s13578-018-0227-1 (2018).

55. Feng, X., Zhou, S., Wang, J. \& Hu, W. microRNA profiles and functions in mosquitoes. PLoS Neglect. Trop. D. 12, e0006463. https:// doi.org/10.1371/journal.pntd.0006463 (2018).

56. Saldaña, M. A. et al. Zika virus alters the microRNA expression profile and elicits an RNAi response in Aedes aegypti mosquitoes. PLoS Neglect. Trop. D. 11, e0005760. https://doi.org/10.1371/journal.pntd.0005760 (2017).

57. Ruby, J. G. et al. Evolution, biogenesis, expression, and target predictions of a substantially expanded set of Drosophila microRNAs. Genome Res. 17, 1850-1864. https://doi.org/10.1101/gr.6597907 (2007).

58. Gleason, R. J., Anand, A., Kai, T. \& Chen, X. Protecting and diversifying the germline. Genetics 208, 435-471. https://doi.org/10. 1534/genetics.117.300208 (2018).

59. Marco, A. Sex-biased expression of microRNAs in Drosophila melanogaster. Open Biol. 4, 140024. https://doi.org/10.1098/rsob. 140024 (2014)

60. Liu, W. et al. Identification and characterization of the expression profile of microRNAs in Anopheles anthropophagus. Parasit. Vectors 7, 159. https://doi.org/10.1186/1756-3305-7-159 (2014).

61. Seong, K. M., Coates, B. S., Kim, D., Hansen, A. K. \& Pittendrigh, B. R. Differentially expressed microRNAs associated with changes of transcript levels in detoxification pathways and DDT-resistance in the Drosophila melanogaster strain 91-R. PLoS ONE 13, e0196518. https://doi.org/10.1371/journal.pone.0196518 (2018).

62. Friedländer, M. R., Mackowiak, S. D., Li, N., Chen, W. \& Rajewsky, N. miRDeep2 accurately identifies known and hundreds of novel microRNA genes in seven animal clades. Nucleic Acids Res. 40, 37-52. https://doi.org/10.1093/nar/gkr688 (2012).

63. Kozomara, A., Birgaoanu, M. \& Griffiths-Jones, S. miRBase: From microRNA sequences to function. Nucleic Acids Res. 47, D155D162. https://doi.org/10.1093/nar/gky1141 (2019).

64. Weibel, S. Metadata: The foundations of resource description. D-lib Magazine [Online]. http://www.dlib.org/dlib/July95/07wei bel.html (1995).

65. R Core Team. R: A Language and Environment for Statistical Computing, Version 3.0.2 (R Foundation for Statistical Computing, 2019).

66. Yu, G., Smith, D., Zhu, H., Guan, Y. \& Lam, T. T. Y. GGTREE: An R package for visualization and annotation of phylogenetic trees with their covariates and other associated data. Methods Ecol. Evol. 8, 28-36. https://doi.org/10.1111/2041-210X.12628 (2017).

67. Wang, L. G. et al. treeio: And R package for phylogenetic tree input and output with richly annotated and associated data. Mol. Biol. Evol. 37, 599-603. https://doi.org/10.1093/molbev/msz240 (2020).

68. Wickham, H., Hester, J., Chang, W., RStudio, R Core Team. Package "devtools" https://CRAN.R-projet.org/package=devtools (2016).

69. Revell, L. J. phytools: An R package for phylogenetic comparative biology (and other things). Methods Ecol. Evol. 3, $217-223$. https://doi.org/10.1111/j.2041-210X.2011.00169.x (2012).

70. Yu, G. ggimage: Use Image in 'ggplot2'. School of Basic Medical Sciences, Southern Medical University. https://guangchuangyu. github.io (2019).

71. Khan, A. \& Mathelier, A. Intervene: A tool for intersection and visualization of multiple gene or genomic region sets. BMC Bioinform. 18, 287. https://doi.org/10.1186/s12859-017-1708-7 (2017).

72. Gehlenborg, N. UpSetR: A More Scalable Alternative to Venn and Euler Diagrams for Visualizing Intersecting Sets. R package version 1.3.3. (2016).

73. Law, C. W. et al. RNA-seq analysis is easy as 1-2-3 with limma, Glimma and edgeR [version 3; peer review: 3 approved]. F1000Research 5, 1408. https://doi.org/10.12688/f1000research.9005.3 (2018).

74. Chen, Y., McCarthy, D., Robinson, M. \& Smyth, G. K. edgeR: Differential expression analysis of digital gene expression data User's Guide. http://www.bioconductor.org/packages/release/bioc/vignettes/edgeR/inst/doc/edgeRUsersGuide.pdf. Accessed 20 Aug 2020 (2014).

75. Galili, T., O'Callaghan, A., Sidi, J. \& Sievert, C. heatmaply: An R package for creating interactive cluster heatmaps for online publishing. Bioinformatics 34, 1600-1602. https://doi.org/10.1093/bioinformatics/btx657 (2018).

76. Su, S. et al. Glimma: Interactive graphics for gene expression analysis. Bioinformatics 33, 2050-2052. https://doi.org/10.1093/bioin formatics/btx094 (2017).

77. Neuwirth, E. RColorBrewer: ColorBrewer palettes. R package version, 1.0-2. https://CRAN.R-projet.org/package=RColorBrewer (2007).

78. Ritchie, M. E. et al. limma powers differential expression analyses for RNA-sequencing and microarray studies. Nucleic Acids Res. 43, e47. https://doi.org/10.1093/nar/gkv007 (2015).

79. Robinson, M. D., McCarthy, D. J. \& Smyth, G. K. edgeR: A bioconductor package for differential expression analysis of digital gene expression data. Bioinformatics 26, 139-140. https://doi.org/10.1093/bioinformatics/btp616 (2010).

\section{Author contributions}

First conceived the idea: I.P.R.S., D.R.S.T., C.Y.Z., G.P.G. Carried out the experiments: I.P.R.S., D.R.S.T., M.L.R.A. Collected the data: G.L., O.K.V.S., M.D.L.L.M.F., I.D.E., L.E.M.V., Y.Z. Conducted the data analysis: M.D.L.L.M.F., 
I.D.E., L.E.M.V., Y.Z., A.E.F.S., X.C., D.R.P., C.Y.Z., G.P.G. Supervised the findings of the work: I.P.R.S., D.R.S.T., G.L., O.K.V.S., M.L.R.A., M.D.L.L.M.F., I.D.E., L.E.M.V., Y.Z., A.E.F.S., X.C., D.R.P., C.Y.Z., G.P.G. Discussed the results: I.P.R.S., D.R.S.T., M.L.G.R., M.A.G.G., G.L., O.K.V.S., M.L.R.A., M.D.L.L.M.F., I.D.E., L.E.M.V., Y.Z., A.E.F.S., X.C., D.R.P., C.Y.Z., G.P.G. Wrote the final manuscript version: I.P.R.S., D.R.S.T., M.L.G.R., G.L., O.K.V.S., M.L.R.A., M.D.L.L.M.F., I.D.E., L.E.M.V., Y.Z., A.E.F.S., X.C., D.R.P., C.Y.Z., G.P.G.

\section{Competing interests}

The authors declare no competing interests.

\section{Additional information}

Supplementary Information The online version contains supplementary material available at https://doi.org/ 10.1038/s41598-021-90095-9.

Correspondence and requests for materials should be addressed to I.P.R.-S. or G.P.-G.

Reprints and permissions information is available at www.nature.com/reprints.

Publisher's note Springer Nature remains neutral with regard to jurisdictional claims in published maps and institutional affiliations.

(c) (i) Open Access This article is licensed under a Creative Commons Attribution 4.0 International License, which permits use, sharing, adaptation, distribution and reproduction in any medium or format, as long as you give appropriate credit to the original author(s) and the source, provide a link to the Creative Commons licence, and indicate if changes were made. The images or other third party material in this article are included in the article's Creative Commons licence, unless indicated otherwise in a credit line to the material. If material is not included in the article's Creative Commons licence and your intended use is not permitted by statutory regulation or exceeds the permitted use, you will need to obtain permission directly from the copyright holder. To view a copy of this licence, visit http://creativecommons.org/licenses/by/4.0/.

(C) The Author(s) 2021 